\title{
FETAL POSTERIOR COMMUNICATING ARTERY AS A CONDUIT FOR CONCURRENT ANTERIOR AND POSTERIOR CIRCULATION INFARCT: A CLINICAL CASE REPORT
}

\author{
Mohamed Azlam Micdhadhu ${ }^{1 *}$, Kho Ko Hin ${ }^{1}$, Mazeda Murad ${ }^{2}$, Irene Looi ${ }^{1}$ \\ ${ }^{1}$ Internal Medicine Department, Hospital Seberang Jaya. \\ ${ }^{2}$ Radiology Department, Hospital Seberang Jaya.
}

\section{*Corresponding Author:}

Dr. Mohamed Azlam Micdhadhu, Internal Medicine Department, Hospital Seberang Jaya, Pulau

Pinang, Malaysia. Tel: +604-3827333

DOI: https://doi.org/10.32896/cvns.v3n4.7-11

Published: 31.12 .2021

Article History: Received October 30, 2020; Revised October 07, 2021; Accepted October 28, 2021

\begin{abstract}
Fetal type posterior cerebral artery (FTPCA) is a variant of posterior circulation of brain, in which the distal part of posterior cerebral artery (PCA) is perfused by a branch of internal carotid artery (ICA) via fetal posterior communicating artery (fetal PCOM). In the presence of fetal PCOM, a paradoxical concurrent infarction of anterior and posterior circulation may happen. We report a 67-year-old man who presented with sudden onset right sided weakness and aphasia, with National Institutes of Health Stroke Scale (NIHSS) score of 22 and clinically diagnosed to have left total anterior circulation infarct (TACI). Subsequently, he received IV Alteplase as a standard hyperacute ischemic stroke treatment. Computed tomography angiography (CTA) of brain showed left FTPCA with prominent left fetal PCOM. Subsequent computed tomography (CT) of brain showed concurrent left middle cerebral artery (MCA) and PCA territories infarct. CTA brain is commonly done in ischemic stroke cases to assess presence of large vessel occlusions and intracranial or extracranial atherosclerotic disease. However, this case depicts its additional role in detecting anatomical variants of cerebral circulation. In terms of clinical importance, presence of multiple territories infarction portends a poorer neurological outcome.
\end{abstract}

Keywords: Infarct

\section{BACKGROUND}

Posterior cerebral artery (PCA) is an important branch of basilar artery in posterior circulation of the brain. It functions to supply the occipital lobe, the inferomedial temporal lobe, and portions of the posterior inferior parietal lobe. [1] Fetal origin of the PCA is a common variant in the posterior cerebral circulation, with an estimated prevalence of $15-32 \%$ of individuals. [2] This common variant is mainly detected only after a patient has suffered an ischemic stroke or when a noninvasive or invasive cerebral angiography is performed for various indications.
Fetal type PCA (FTPCA) denotes bulk of blood supply to PCA territory arising from internal carotid artery (ICA) via posterior communicating artery (PCOM) with absent or hypoplastic P1 segment of PCA. This variant of PCOM is called fetal PCOM. Van Raamt et al. have proposed the term full fetal type PCA (full FTPCA) for total absence of P1 segment, while partial FTPCA for hypoplastic P1 segment. In both circumstances, the posterior communicating artery appears larger than usual on CTA brain. [3] Although FTPCA is a normal variant; it results in the lack of proper collaterals and larger than usual posterior communicating artery may pose an 
increased risk for ischemic injury to the anterior as well as posterior cerebral regions. [4] A concurrent infarct involving multiple arterial territories herald a poorer neurological outcome. We report a case of concurrent left middle cerebral artery (MCA) and PCA territory infarction, sparing the anterior cerebral artery (ACA).

\section{CASE PRESENTATION}

A 67-year-old man presented with sudden onset of right sided body weakness and slurred speech at 11.30am. Otherwise, he didn't complain of headache, blurred vision, vomiting or numbness. There was no seizure. He neither complained of chest pain nor palpitation. He had underlying schizophrenia diagnosed in 2015, on chlorpromazine $100 \mathrm{mg}$ on night. He had a laparotomy 3 years ago, which the cause remained uncertain. He is an active smoker but not known to have diabetes mellitus, hypertension or dyslipidaemia.

Upon arrival, his Glasgow Coma Scale (GCS) was E4V3M5, pupils bilaterally equal and reactive. Initial blood pressure $138 / 87 \mathrm{mmHg}$,

\section{Non contrasted CT brain showed as below:}

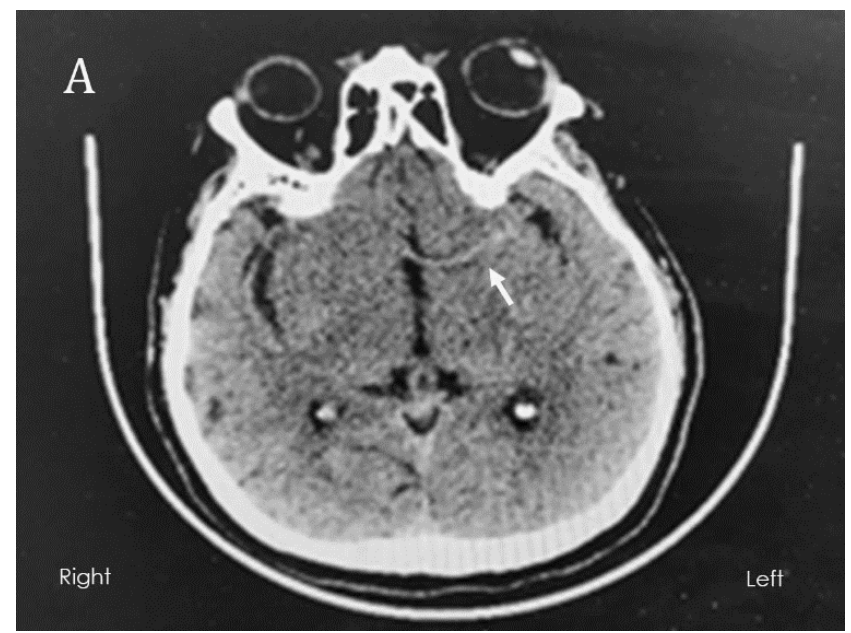

Figure A. Hyperdense left MCA sign. (arrow) pulse rate 57 beats per minute, respiratory rate 16 breaths per minute, temperature $37^{\circ} \mathrm{C}$, pain score 0 . His oxygen saturation was $95 \%$, and capillary blood glucose $5.7 \mathrm{mmol} / \mathrm{L}$. His lungs were clear on auscultation, no murmur and no carotid bruit. Examination of abdomen revealed a midline laparotomy scar. Neurological assessment revealed normal tone with right upper limb and lower limb muscle power grading $5 / 5$, while left upper limb and lower limb power $2 / 5$ based on medical research council scale for muscle strength. Otherwise, reflexes were normal with extensor plantar response bilaterally.

His initial NIHSS score was 22. Electrocardiography revealed sinus rhythm. Blood investigations revealed hemoglobin count $16.2 \mathrm{~g} / \mathrm{dL}$, white blood cell count $13 \times 10^{3} / \mu \mathrm{L}$, platelet count $269 \times 10^{3} / \mu \mathrm{L}$, hematocrit $48 \%$, sodium $141 \mathrm{mmol} / \mathrm{L}$, potassium $4 \mathrm{mmol} / \mathrm{L}$, urea $4.1 \mathrm{mmol} / \mathrm{L}$, creatinine $105 \mu \mathrm{mol} / \mathrm{L}$, corrected calcium $2.07 \mathrm{mmol} / \mathrm{L}$, phosphate $1.43 \mathrm{mmol} / \mathrm{L}$, prothrombin time $13 \mathrm{sec}$, activated plasma thromboplastin time $41 \mathrm{sec}$, international normalised ratio (INR) 0.99 .

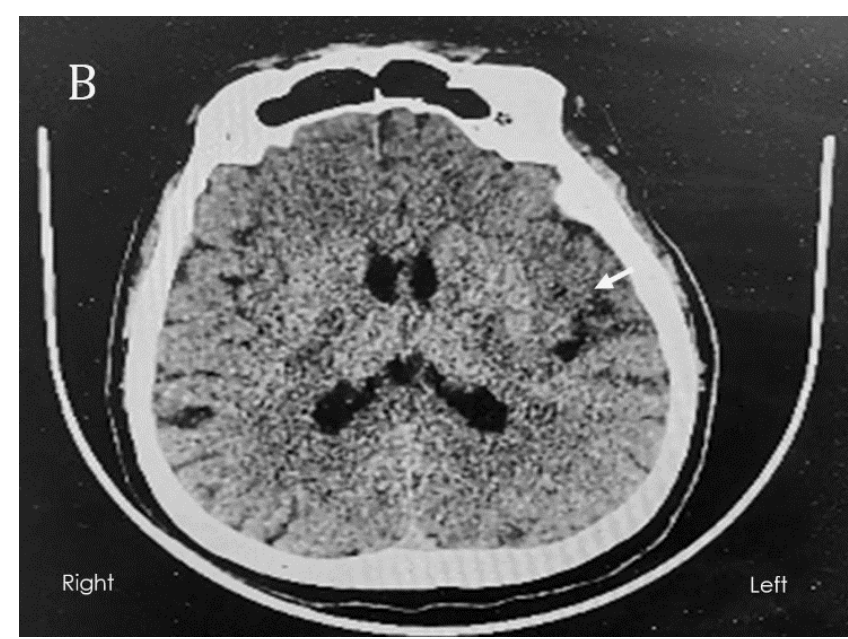

Figure B. Hypodense of left parietal and insular cortex. (arrow) 


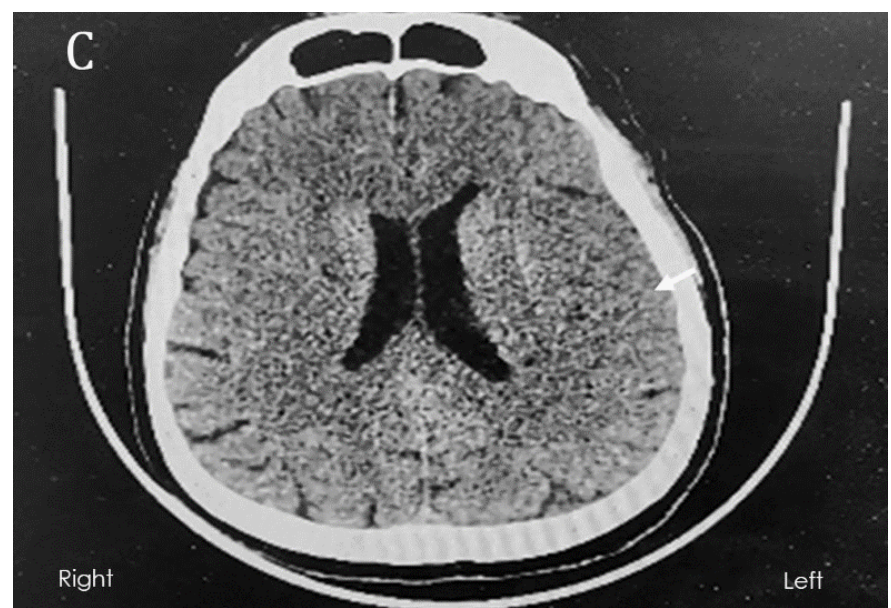

Figure C. Loss of sulci over left parietal area. (arrow)
The ASPECT score was 5, involving left M1, M2, M3, M4, and insular cortex.

Based on clinical symptoms and imaging, he was diagnosed as left total anterior circulation infarct (TACI) involving left MCA territory, based on Oxfordshire Community Stroke Project (OCSP) classification. Intravenous Alteplase $50 \mathrm{mg}$ (based on body weight) was given at 3hour 20minutes from symptom onset.

\section{Subsequently, a cerebral CT angiogram was performed. The imaging as follows:}
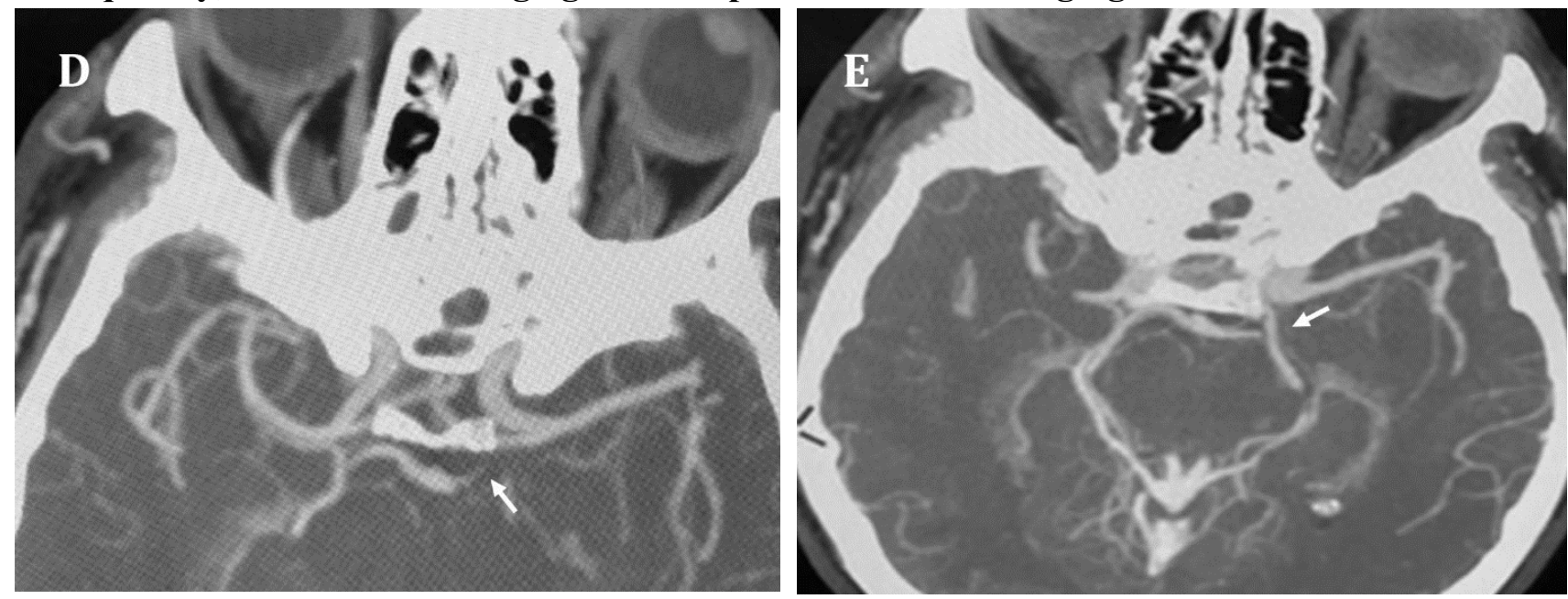

Figure D: Hypoplastic left P1. (arrow)

Bilateral MCA and ACA were patent. Intracranial part of bilateral ICA was patent. However, unable to comment on extracranial ICA as our CT angiography was not extended down to arch of aorta.

He was admitted into intensive care unit for close monitoring. Systolic blood pressure remained in the range of 150-180 during and after alteplase infusion. Unfortunately, 2 hours later he developed respiratory distress due to acute pulmonary oedema. He was intubated and ventilated, after initial intravenous frusemide failed to improve his clinical state. 


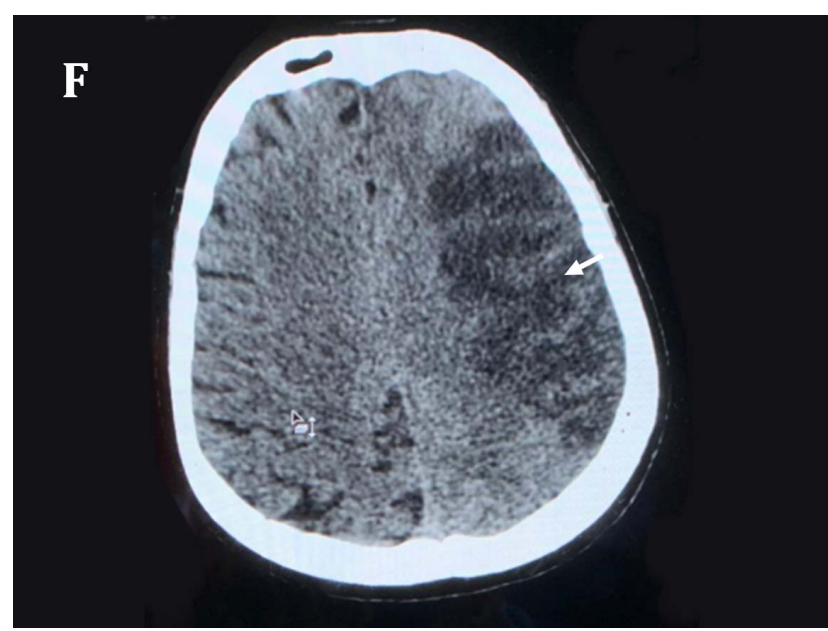

Figure F: Infarct over left MCA territory. (arrow)

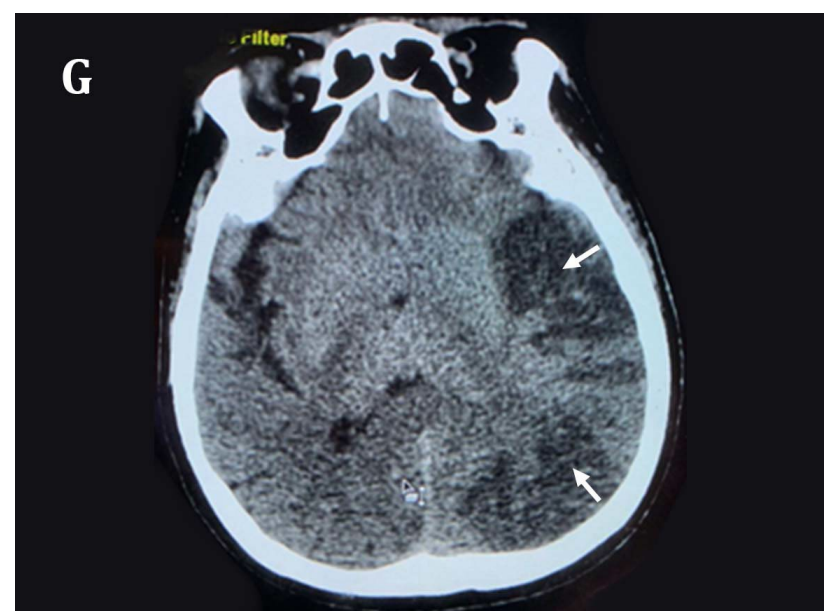

Figure H: Infarct over both left MCA (arrow) and PCA territory. (block arrow)

\section{DISCUSSION}

MCA infarct is one of the devastating forms of stroke. Clinically they can come in the form of Partial Anterior Circulation Infarct (PACI) or Total Anterior Circulation Infarct (TACI). Our patient presented as TACI, however to our surprise he had a concurrent MCA and PCA territory infarcts.

A fetal origin of Posterior Cerebral Artery is a common variant in posterior circulation, estimated to be of $15-32 \%$ of population. This means the PCOM will be larger than P1 segment of PCA, which the latter will be hypoplastic or at times absent. Hence, bulk of blood supply to PCA comes via PCOM, from ipsilateral ICA. This type of PCOM is named fetal PCOM.

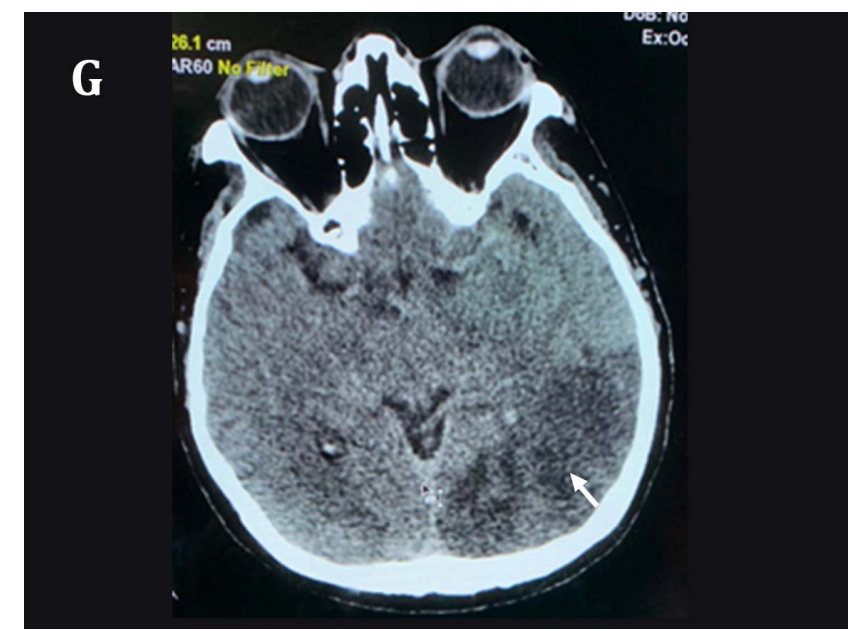

Figure G: Infarct over left PCA territory. (block arrow)

Repeated CT brain at day 4 of stroke revealed well established concurrent right MCA and PCA territory infarctions. Ipsilateral mild cerebral oedema was present as well. Hence, IV mannitol $20 \%$ 200cc 12-hourly was given for one day. His Glasgow Coma Scale (GCS) was E2VTM5 at day 5 of stroke despite 24 hours without sedation. Subcutaneous heparin 5000unit BD was started for deep vein thrombosis prophylaxis and oral aspirin was initiated at day 5 of stroke. He was planned for trachaeostomy and weaning off ventilatory support. However, he developed pneumonia and acute kidney injury requiring high ventilator setting and hemodialysis respectively. Eventually, succumbed to sepsis on day 13 of stroke.

Our patient suffered a concurrent left MCA and PCA territory infarcts due to the presence of left fetal PCOM (fetal origin of PCA). We postulate an artery-to-arteries embolization (from extracranial part of left internal carotid to left PCA-P2 segment and left MCA).

Nevertheless, CT angiography did not show acute thrombus in either vessel, which could be due to lysed clot (post intravenous thrombolysis) or the clots could have migrated further distally. Distal occlusions are not readily visible on CT angiography.

Otherwise, a concurrent infarct could be as well of cardiac origin, whereby a thrombus from intracardiac region could have travelled up to intracranial artery via internal carotid, causing occlusion of left MCA and PCA, with left PCOM 
serving as a conduit for cross embolization from the ICA to the PCA P2 segment or its distal branches. Unfortunately, 24 hours cardiac activity recording (Holter examination) and echocardiography was not done to confirm possibility of cardioembolism.

According to van Raamt et al, a full FTPCA carries a higher vascular insufficiency risk than a partial FTPCA because leptomeningeal anastomoses do not form between the anterior and posterior circulation if a person has a full FTPCA.[3] A series of cases reported by Nico et.al showed patients with fetal origin of PCA is more likely to have a posterior circulation infarct, rather than concurrent anterior and posterior circulation infarct [5]. This is supported by a theory of increased ICA-PCA pressure gradient due to hemodynamic changes from stenotic lesions in vertebrobasilar system or significant artherosclerosis of proximal ICA.[6]

This case of fetal-type PCA (FTPCA) and concurrent PCA-MCA territory infarction illustrates the variability in anatomy and clinical significance of PCA variants. FTPCA is clearly

\section{REFERENCES}

1. Chaves C, Caplan LR. Posterior cerebral artery. In: Caplan LR, van Gijn J, eds. Stroke Syndromes. 3rd ed. Cambridge, England: Cambridge University Press, 2012. p. 405-418.

2. Amir Shaban, Karen C. Albright, Amelia K. Boehme, Sheryl Martin-Schild. Circle of Willis Variants: Fetal PCA. Stroke

Research and Treatment, 2013. https://doi.org/10.1155/2013/105937

3. Van Raamt AF, Mali WP, van Laar PJ, van der Graaf Y. The fetal variant of the circle of Willis and its influence on the cerebral collateral circulation. Cerebrovasc Dis, 2006. 22(4): p. 217-224. the mechanism of paradoxical infarction in this patient. To our knowledge, reports of concurrent multiple territories infarct are rare and adds on significantly to poor outcome of major acute ischemic strokes (TACI and PACI). This case illustrates a typical presentation of TACI and subsequent hyperacute stroke reperfusion therapy as the treatment for TACI. Also, this highlights the importance of CT angiography of brain in aiding management of acute ischemic stroke. Furthermore, it has served to explain why patient developed concurrent multiple territory infarcts. FTPCA can increase the extent and severity of anterior circulation strokes by allowing additional infarction in the PCA territory. Whether FTPCA increases the overall risk of stroke independent of other risk factors is not clear. The optimal stroke prevention regimen for individuals with FTPCA and one or more stroke risk factors is also unclear. Risk factors control remains the mainstay in stroke prevention.

The authors acknowledge the generosity of Mr M's family in providing permission to write about Mr M's treatment in our facility.

4. Arjal, R. K., et al., The study of fetal-type posterior cerebral circulation on multislice $\mathrm{CT}$ angiography and its influence on cerebral ischemic strokes Clin Imaging, 2014. 38(3): p. 221-225. https://doi.org/10.1016/j.clinimag.2014.01. 007

5. Nico S, Timo K, Marta K, et al. Fetal-type posterior cerebral artery: a not so benign anatomical variant. Stroke, 2020. 51(1): p. 225.

6. Lambert, S.L., et al., Fetal-Type Variants of the Posterior Cerebral Artery and Concurrent Infarction in the Major Arterial Territories of the Cerebral Hemisphere. J Investig Med High Impact Case Rep, 2016. 4(3): p. 2324709616665409. https://doi.org/10.1177/2324709616665409 hic.sagepub.com 